\title{
On the Signatures of Torus Knots
}

by

\section{Maciej BORODZIK and Krzysztof OLESZKIEWICZ}

Presented by Andrzej BIAŁYNICKI-BIRULA

Summary. We study properties of the signature function of the torus knot $T_{p, q}$. First we provide a very elementary proof of the formula for the integral of the signature over the circle. We also obtain a closed formula for the Tristram-Levine signature of a torus knot in terms of Dedekind sums.

1. Preliminaries. Let $K$ be a knot in $S^{3}$ with a Seifert matrix $S$. Let also $z \in S^{1}, z \neq 1$, be a complex number. The Tristram-Levine signature $\sigma(z)$ is the signature of the hermitian form

$$
(1-z) S+(1-\bar{z}) S^{T}
$$

This is obviously an integer-valued piecewise constant function. It does not depend on the particular choice of Seifert matrix. For $z=-1$ we get an invariant $\sigma_{\text {ord }}$, which is called the (ordinary) signature. We also define the integral

$$
I_{K}=\int_{0}^{1} \sigma\left(e^{2 \pi i x}\right) d x .
$$

Signatures are very strong knot cobordism invariants, which can be used to bound the four-genus and the unknotting number of $K$. The integral $I_{K}$ of the signature function is one of the so called $\rho$ invariants of knots (see COT1, COT2 2) and is of independent interest.

For a torus knot $T_{p, q}$, where $\operatorname{gcd}(p, q)=1$, the signature function can be expressed in the following nice way (see [Li] or [Kau, Chapter XII]): 
Proposition 1.1. Let

$$
\Sigma=\left\{\frac{k}{p}+\frac{l}{q}: 1 \leq k \leq p-1,1 \leq l \leq q-1\right\} .
$$

Then for any $x \in(0,1) \backslash \Sigma$ we have

$$
\sigma\left(e^{2 \pi i x}\right)=|\Sigma \backslash(x, x+1)|-|\Sigma \cap(x, x+1)|,
$$

where $|\cdot|$ denotes cardinality. In particular

$$
\sigma_{\text {ord }}=|\Sigma \backslash(1 / 2,3 / 2)|-|\Sigma \cap(1 / 2,3 / 2)| .
$$

Explicit formulae for $\sigma_{\text {ord }}$ and $I_{K}$ of torus knots have been known in the literature for quite a long time. In fact, by a result of Viro (see (2.4)) $\sigma_{\text {ord }}$ is equal to $\tau_{2}$, which was computed in [HZ for $p$ and $q$ odd, and (denoted as $\left.\sigma\left(f+z^{2}\right)\right)$ in Nem in the general case. On the other hand, Kirby and Melvin [KM, Remark 3.9] and [Nem, Example 4.3] provided a formula for $I_{K}$. Nevertheless, all the above-mentioned results are related more to singularity theory and low-dimensional topology than to knot theory itself.

After the discovery of $\rho$ invariants, the interest in computing $I_{K}$ for various families of knots grew significantly. Two independent new proofs of the formula for $I_{K}$ of torus knots [Bo, $\mathrm{Co}$ ] appeared in 2009. In particular [Bo] provided a bridge between the $I_{K}$ and invariants of cuspidal singularities of complex plane curves.

In this paper we present an elementary proof of the formula for $I_{K}$ (Proposition 2.1). We also cite a formula of Némethi and draw some consequences from it. In Section 4 we use a theorem of Rosen to obtain the explicit value of the signature $\sigma(z)$ of a torus knot not only for $z=-1$, but also for almost every $z \in S^{1} \backslash\{1\}$ (Proposition 4.3). This result seems to be new. In Section 5 we show that the formula for $\sigma_{\text {ord }}\left(T_{p, q}\right)$ cannot be written as a rational function of $p$ and $q$.

\section{Formula for the integral}

Proposition 2.1. For a torus knot $T_{p, q}$ we have

$$
I=-\frac{1}{3}\left(p-\frac{1}{p}\right)\left(q-\frac{1}{q}\right) .
$$

This proposition was first proved in [KM], Remark 3.9]. We refer to [Nem, Bo, $\mathrm{Co}$ for other proofs.

Proof. Let $f(x)=-\sigma\left(e^{2 \pi i x}\right)$ and $J=\int_{0}^{1} f(x) d x=-I$. Then

$$
f(x)=\sum_{y \in \Sigma} \mathbf{1}_{(x, x+1)}(y)-\sum_{y \in \Sigma} \mathbf{1}_{\mathbb{R} \backslash(x, x+1)}(y) .
$$


(Here, for $A \subset \mathbb{R}, \mathbf{1}_{A}$ denotes the function which is equal to 1 on $A$ and 0 away from $A$.) Hence

$$
J=\sum_{y \in \Sigma} \int_{0}^{1}\left(\mathbf{1}_{(y-1, y)}(x)-\mathbf{1}_{\mathbb{R} \backslash(y-1, y)}(x)\right) d x=\sum_{y \in \Sigma}(1-2|y-1|) .
$$

It follows that

$$
J=\sum_{k=1}^{p-1} \sum_{l=1}^{q-1}\left(1-2\left|\frac{k}{p}+\frac{l}{q}-1\right|\right) .
$$

As for any $u, v \in \mathbb{R}$ we have

$$
1-2|u+v-1|=2 \min (1-u, v)+2 \min (u, 1-v)-1,
$$

it follows that

$$
\begin{aligned}
J & =2 \sum_{k=1}^{p-1} \sum_{l=1}^{q-1} \min \left(\frac{p-k}{p}, \frac{l}{q}\right)+2 \sum_{k=1}^{p-1} \sum_{l=1}^{q-1} \min \left(\frac{k}{p}, \frac{q-l}{q}\right)-(p-1)(q-1) \\
& =4 \sum_{k=1}^{p-1} \sum_{l=1}^{q-1} \min \left(\frac{k}{p}, \frac{l}{q}\right)-(p-1)(q-1) \\
& =\frac{4}{p q} \sum_{k=1}^{p-1} \sum_{l=1}^{q-1} \min (q k, p l)-(p-1)(q-1) .
\end{aligned}
$$

Now, obviously,

$$
\begin{aligned}
& \sum_{k=1}^{p-1} \sum_{l=1}^{q-1} \min (q k, p l) \\
& \quad=\sum_{s=0}^{\infty} \mid\{(k, l) \in\{1, \ldots, p-1\} \times\{1, \ldots, q-1\}: q k>s \text { and } p l>s\} \mid \\
& \quad=\sum_{s=0}^{p q-1}(p-1-\lfloor s / q\rfloor)(q-1-\lfloor s / p\rfloor) .
\end{aligned}
$$

We can multiply the expressions in parentheses. Then, as

$$
\sum_{s=0}^{p q-1}\lfloor s / p\rfloor=p \sum_{l=0}^{q-1} l=\frac{1}{2} p q(q-1),
$$


we get

$$
\begin{aligned}
& \sum_{s=0}^{p q-1}(p-1-\lfloor s / q\rfloor)(q-1-\lfloor s / p\rfloor) \\
& =p q(p-1)(q-1)-\frac{1}{2} p q(p-1)(q-1)-\frac{1}{2} p q(p-1)(q-1)+\sum_{s=0}^{p q-1}\lfloor s / p\rfloor\lfloor s / q\rfloor \\
& \left.=\sum_{s=0}^{p q-1}\lfloor s / p\rfloor \leq s / q\right\rfloor .
\end{aligned}
$$

It remains to compute the last sum. To this end denote by $R_{p}(s)$ the remainder of $s$ modulo $p$. Then

$$
\begin{aligned}
\sum_{s=0}^{p q-1}\lfloor s / p\rfloor\lfloor s / q\rfloor & =\sum_{s=0}^{p q-1}\left(\frac{s-R_{p}(s)}{p} \cdot \frac{s-R_{q}(s)}{q}\right) \\
& =\frac{1}{p q}\left(\sum_{s=0}^{p q-1} s^{2}-\sum_{s=0}^{p q-1} s R_{p}(s)-\sum_{s=0}^{p q-1} s R_{q}(s)+\sum_{s=0}^{p q-1} R_{p}(s) R_{q}(s)\right) \\
& =\frac{1}{3} p^{2} q^{2}+\frac{1}{4} p q-\frac{1}{4} p^{2} q-\frac{1}{4} p q^{2}-\frac{1}{12} p^{2}-\frac{1}{12} q^{2}+\frac{1}{12},
\end{aligned}
$$

where we used the fact that

$$
\sum_{s=0}^{p q-1} R_{p}(s) R_{q}(s)=\sum_{k=0}^{p-1} \sum_{l=0}^{q-1} k l
$$

by the Chinese remainder theorem.

Putting all the pieces together we obtain the desired formula.

Let us now present another proof, due to Némethi Nem (see also Br. [HZ]). Before we do this, we recall some facts from topology.

Assume that the knot $K$ is drawn on $S^{3}=\partial B^{4}$ and consider a Seifert surface $F$ of $K$. Let us push it slightly into $B^{4}$ and, for an integer $m$, let $N_{m}$ be the $m$-fold cyclic cover of $B^{4}$ branched along $F$. Then the quantity $\tau_{m}=\sigma\left(N_{m}\right)$ (here $\sigma$ is the signature of a four-manifold with boundary) is independent of the choices made. We have the following formula essentially due to Viro (see [GLM, Section 2] or [Vi]):

$$
\tau_{m}=\sum_{k=1}^{m-1} \sigma_{K}\left(\xi^{k}\right),
$$

where $\xi$ is a primitive root of unity of order $m$. In particular, since $\sigma$ is a 
Riemann integrable function, we have

$$
I=\int_{0}^{1} \sigma\left(e^{2 \pi i x}\right) d x=\lim _{m \rightarrow \infty} \frac{1}{m} \tau_{m} .
$$

On the other hand

$$
\tau_{2}(K)=\sigma_{\text {ord }}(K) .
$$

If $K$ is a torus knot $T_{p, q}$ and $m, p, q$ are pairwise coprime, then the $m$-fold cover of $S^{3}$ branched along $K$ is diffeomorphic to the Brieskorn homology sphere $B(p, q, m)$ (see [Br], GLM, Section 5]). Then $\tau_{m}$ turns out [HZ, Sections 10.2 and 11] to be the signature of the manifold $X_{p, q, m}$ defined as the intersection of $z_{1}^{p}+z_{2}^{q}+z_{3}^{m}=\varepsilon$ with $B(0,1) \subset \mathbb{C}^{3}$. In this context $\tau_{m}$ was computed in [HZ, Formula (11), p. 122] and [Nem, Example 4.3]. Especially the last formula is worth citing (Némethi uses $m(S(f))$ to denote the limit (2.3)):

$$
I=-4(s(p, q)+s(q, p)+s(1, p q)) .
$$

Here $s(a, b)$ is the Dedekind sum (see Section 3). As by elementary computations

$$
s(1, p q)=\frac{(p q-1)(p q-2)}{12 p q},
$$

we get

$$
s(p, q)+s(q, p)=-\frac{I}{4}-\frac{(p q-1)(p q-2)}{12 p q} .
$$

Now we can view the above equation as defining $I$ in terms of $s(p, q)+s(q, p)$; but if we know $I$, we know $s(p, q)+s(q, p)$. In other words we get the following observation.

Corollary 2.2. Any proof of Proposition 2.1 which does not involve Dedekind sums provides a proof of the Dedekind reciprocity law.

3. Lattice points in the triangle. Let us recall basic definitions. For a real number $x,\lfloor x\rfloor$ denotes the integer part and $\{x\}=x-\lfloor x\rfloor$ the fractional part. The sawtooth function is defined by

$$
\langle x\rangle= \begin{cases}\{x\}-1 / 2, & x \notin \mathbb{Z}, \\ 0, & x \in \mathbb{Z} .\end{cases}
$$

Sometimes $\langle x\rangle$ is denoted $((x))$. We prefer the former notation because it does not lead to confusion with ordinary parentheses. We can now define the 
following functions (below $p, q$ and $m$ are integers and $x, y$ are real numbers):

$$
\begin{aligned}
s(p, q) & =\sum_{j=0}^{p-1}\left\langle\frac{j}{q}\right\rangle\left\langle\frac{p j}{q}\right\rangle, \\
s(p, q ; x, y) & =\sum_{j=0}^{p-1}\left\langle\frac{j+y}{q}\right\rangle\left\langle p \frac{j+y}{q}+x\right\rangle .
\end{aligned}
$$

$s(p, q)$ is called the (ordinary) Dedekind sum, while $s(p, q ; x, y)$ is a generalized Dedekind sum. These functions satisfy the following reciprocity laws (see [RG, $\mathrm{HZ}]$ ). If $m, p$ and $q$ are pairwise coprime, then

$$
\begin{aligned}
s(p, q)+s(q, p) & =\frac{1}{12}\left(\frac{p}{q}+\frac{q}{p}+\frac{1}{p q}\right)-\frac{1}{4} \\
s(p, q ; x, y)+s(q, p ; y, x) & =-\frac{1}{4} d(x) d(y)+\langle x\rangle\langle y\rangle \\
& +\frac{1}{2}\left(\frac{q}{p} \Psi_{2}(y)+\frac{1}{p q} \Psi_{2}(p y+q x)+\frac{p}{q} \Psi_{2}(x)\right) .
\end{aligned}
$$

Here

$$
d(x)= \begin{cases}1 & \text { if } x \in \mathbb{Z} \\ 0 & \text { otherwise }\end{cases}
$$

and

$$
\Psi_{2}(x)=B_{2}(\{x\})=\{x\}^{2}-\{x\}+\frac{1}{6}
$$

is the second Bernoulli polynomial. Now for a fixed $C \in[0,1)$ and $p, q$ coprime, let

$$
A(p, q ; C)=\left\{(k, l) \in \mathbb{Z}_{\geq 0}^{2}: 0 \leq \frac{k}{p}+\frac{l}{q}<1-C\right\}
$$

and

$$
N(p, q ; C)=|A(p, q ; C)| .
$$

We have the following result due to Rosen [Ro, Theorem 3.4].

Proposition 3.1. In this case

$$
\begin{aligned}
N(p, q ; C)= & \frac{(1-C)^{2}}{2} p q+\frac{1-C}{2}(p+q)+\frac{q}{12 p}+\frac{p}{12 q}+K \\
& -s(p, q ; C p, 0)-s(q, p ; C q, 0)+\langle C p\rangle+\langle C q\rangle \\
& +(1-C)\langle C p q\rangle-\left(\frac{7}{8} \delta_{0}+\frac{3}{8} \delta_{1}-\frac{1}{8} \delta_{2}\right)+\frac{1}{4},
\end{aligned}
$$


where

$$
K= \begin{cases}\frac{1}{12 p q}-\frac{1}{8} & \text { if } C p q \in \mathbb{Z}, \\ \frac{1}{2 p q} \Psi_{2}(C p q) & \text { otherwise }\end{cases}
$$

and for $r=0,1,2, \delta_{r}$ is the number of non-negative integers $k, l$ such that $k / p+l / q+C=r$.

This proposition has an important corollary [Ro, Corollary 3.5].

COROLlary 3.2. If $p$ and $q$ are odd and coprime, then

$$
N\left(p, q ; \frac{1}{2}\right)=\frac{p q}{8}+\frac{p+q}{4}+\frac{q}{6 p}+\frac{p}{6 q}+\frac{1}{24 p q}-s(2 p, q)-s(2 q, p) .
$$

If $p$ and $q$ are coprime and $q$ is even, then

$$
N\left(p, q ; \frac{1}{2}\right)=\frac{p q}{8}+\frac{p+q}{4}-s(2 p, q)+2 s(p, q) .
$$

We shall use these results to compute the signature of the torus knots. We need the following trivial lemma:

Lemma 3.3. The number of points $(k, l) \in A(p, q ; C)$ such that $k l=0$ is equal to

$$
Z(p, q ; C)=\lfloor(1-C) p\rfloor+\lfloor(1-C) q\rfloor+1-d((1-C) p)-d((1-C) q),
$$

where $d(x)$ is again 1 if $x \in \mathbb{Z}$, and 0 otherwise.

If $C p$ and $C q$ are not integers, Lemma 3.3 says that

$$
Z(p, q ; C)=(1-C)(p+q)-\langle(1-C) p\rangle-\langle(1-C) q\rangle .
$$

4. Explicit formulae for the signatures. We begin by computing the value of the ordinary signature. As already mentioned, $\sigma_{\text {ord }}=\tau_{2}$ (see (2.4) $)$ so the first result below is in general known $[\mathrm{HZ}$, $\mathrm{Nem}$, but not necessarily in the context of knot theory.

Proposition 4.1. If $p$ and $q$ are both odd and coprime, then the ordinary signature of the torus knot $T_{p, q}$ satisfies

$$
\sigma_{\text {ord }}\left(T_{p, q}\right)=-\frac{p q}{2}+\frac{2 p}{3 q}+\frac{2 q}{3 p}+\frac{1}{6 p q}-4(s(2 p, q)+s(2 q, p))-1,
$$

where $s(x, y)$ is the Dedekind sum (see Section 3 or [RG]; cf. [HZ, Formula (11), p. 122]). If $p$ is odd and $q>2$ is even, then

$$
\sigma_{\text {ord }}\left(T_{p, q}\right)=-\frac{p q}{2}+1+4 s(2 p, q)-8 s(p, q) .
$$

Proof. Let $\Sigma$ be as in 1.1). We can write $\sigma_{\text {ord }}$ as

$$
\sigma_{\text {ord }}=4|\Sigma \cap(0,1 / 2)|-|\Sigma| \text {. }
$$


Since $|\Sigma|=(p-1)(q-1)$, we need to find a closed formula for

$$
\begin{aligned}
S(p, q) & =|\Sigma \cap(0,1 / 2)| \\
& =\left|\left\{x=\frac{k}{p}+\frac{l}{q}: x<\frac{1}{2}, 1 \leq k \leq p-1,1 \leq l \leq q-1\right\}\right| .
\end{aligned}
$$

From the definition we get immediately

$$
S(p, q)=N(p, q ; 1 / 2)-Z(p, q ; 1 / 2) .
$$

Now $Z(p, q ; 1 / 2)=\frac{1}{2}(p+q)$ if $p$ and $q$ are both odd, and $\frac{1}{2}(p+q-1)$ if $q$ is even and $q>2$. Hence, for $p$ and $q$ odd we have, by (3.4),

$$
S(p, q)=\frac{p q}{8}-\frac{p+q}{4}-s(2 p, q)+2 s(p, q),
$$

while for $q$ even we have, by 3.5 ,

$$
S(p, q)=\frac{p q}{8}-\frac{p+q}{4}+\frac{1}{2}-s(2 p, q)+2 s(p, q),
$$

and using 4.1 we complete the proof.

REMARK 4.2. Formula 4.2 can be rewritten as

$$
S(p, q)=\sum_{k=1}^{\lfloor p / 2\rfloor}\left\lfloor\frac{q p-2 k q}{2 p}\right\rfloor,
$$

which gives a formula for $\sigma_{\text {ord }}$ using ordinary sums, not Dedekind sums. On the other hand [NY, Proposition 2.1] provides a different formula for $\sigma_{\text {ord }}$ using ordinary sums. The latter is especially useful for providing explicit formulae for $\sigma_{\text {ord }}\left(T_{p, p+r}\right)$ for small values of $r$.

To express explicitly the values of Tristram-Levine signatures at other points let us assume that $C p q$ is not an integer (in particular $C \notin \Sigma$ ). Define

$$
\begin{aligned}
M(p, q ; C)= & N(p, q ; C)-Z(p, q ; C) \\
= & \frac{(1-C)^{2}}{2} p q-\frac{1-C}{2}(p+q) \\
& +\frac{q}{12 p}+\frac{p}{12 q}-s(p, q ; C p, 0)-s(q, p ; C q, 0)+\frac{1}{4} \\
& -\frac{1}{2}(\langle C p\rangle+\langle C q\rangle)+(1-C)\langle C p q\rangle+\frac{1}{2 p q} \Psi_{2}(C p q) .
\end{aligned}
$$

Now it is a trivial consequence of Proposition 1.1 that if $C \in[0,1)$ and $e^{2 \pi i C}=z$, then

$$
\sigma(z)=-(p-1)(q-1)+2 M(p, q ; C)+2 M(p, q ; 1-C) .
$$

Since for any integer $k$ and real $x$ we have $\langle(1-x) k\rangle+\langle x k\rangle=0$, the formula 
for $M(p, q ; C)+M(p, q ; 1-C)$ can be simplified to

$$
\begin{gathered}
\frac{1-2 C+2 C^{2}}{2} p q-\frac{1}{2}(p+q)+\frac{q}{6 p}+\frac{p}{6 q}+(1-2 C)\langle C p q\rangle+\frac{1}{p q}\left(\langle C p q\rangle^{2}-\frac{1}{12}\right)+\frac{1}{2} \\
-s(p, q ; C p, 0)-s(q, p ; C q, 0)-s(p, q ;(1-C) p, 0)-s(q, p ;(1-C) q, 0) .
\end{gathered}
$$

Hence we obtain the following result.

Proposition 4.3. If $z=e^{2 \pi i C}$ where $C \in[0,1)$ is such that $C p q$ is not an integer, then the signature of the torus knot $T_{p, q}$ can be given by the following formula:

$$
\begin{aligned}
& \sigma(z)=-2\left(C-C^{2}\right) p q+\frac{q}{3 p}+\frac{p}{3 q}+(2-4 C)\langle C p q\rangle+\frac{2}{p q}\left(\langle C p q\rangle^{2}-\frac{1}{12}\right) \\
& -2(s(p, q ; C p, 0)+s(q, p ; C q, 0)+s(p, q ;(1-C) p, 0)+s(q, p ;(1-C) q, 0)) .
\end{aligned}
$$

In particular we see rigorously that for large $p$ and $q$ the shape of the function $\sigma\left(e^{2 \pi i x}\right)$ resembles that of the function $2 p q\left(x^{2}-x\right)$.

REMARK 4.4. We can integrate the above formula over the interval $[0,1]$ with respect to $C$. The term $-2\left(C-C^{2}\right) p q$ contributes $-p q / 3$, and the next two terms contribute $q / 3 p$ and $p / 3 q$, respectively. A straightforward computation gives

$$
\int_{0}^{1} C\langle C p q\rangle d C=\frac{1}{12 p q} \text { and } \int_{0}^{1}\left(\langle C p q\rangle^{2}-\frac{1}{12}\right) d C=0 .
$$

All other integrals trivially vanish. We thus recover formula (2.1) from Proposition 4.3 .

\section{Expressing $\sigma_{\text {ord }}\left(T_{p, q}\right)$ as a rational function}

Proposition 5.1. There does not exist a rational function $R(p, q)$ such that for all odd and coprime positive integers $p, q$,

$$
R(p, q)=\sigma_{\text {ord }}\left(T_{p, q}\right) .
$$

Proof. Assume the contrary. Then $S(p, q)=\frac{1}{4}(R(p, q)+(p-1)(q-1))$ is also a rational function.

If $p \mid(q-1)$ and $p, q$ are both odd, the value of $S(p, q)$ can be easily computed using (4.3):

$$
\begin{aligned}
S(p, q) & =\sum_{k=1}^{(p-1) / 2}\left\lfloor\frac{q}{2}-\frac{q k}{p}\right\rfloor=\sum_{k=1}^{(p-1) / 2}\left\lfloor\frac{q-1}{2}-\frac{(q-1) k}{p}+\frac{p-2 k}{2 p}\right\rfloor \\
& =\sum_{k=1}^{(p-1) / 2}\left(\frac{q-1}{2}-k \frac{q-1}{p}\right)=\frac{(q-1)(p-1)^{2}}{8 p} .
\end{aligned}
$$


Since for infinitely many values $(p, q)$ with $q=n p+1$ with $p$ odd and $n$ even, we have $p \mid(q-1)$, it follows that

$$
S(p, q)=\frac{(q-1)(p-1)^{2}}{8 p}
$$

on each line $q=n p+1$. Since these rational functions agree on infinitely many lines, they must be equal.

Now assume that $p=n q+1$ for some even $n$. Similar arguments to those above show that $S(p, q)$ must also be identical to the function

$$
\frac{(p-1)(q-1)^{2}}{8 q} .
$$

This is a contradiction, since these two rational functions are different.

REMARK 5.2. We can also compute values of $S(p, q)$ in many other cases, like $q=n p-1, q=p+2$. With more care we can prove that e.g. $S(p, q)-\lfloor q / p\rfloor$ is not a rational function.

The proof carries over to show that no such rational function exists for the case $p$ even and $q$ odd. We leave the obvious details to the reader.

Acknowledgements. The authors wish to thank Andrew Ranicki for many remarks during the preparation of the paper, and Julia Collins, Stefan Friedl and András Némethi for their interest and comments concerning this article.

The first author is supported by Polish MNiSzW Grant No N N201 397937 and also by the Foundation for Polish Science FNP. The second author is supported by Polish MNiSzW Grant N N201 397437.

\section{References}

[Bo] M. Borodzik, A $\rho$ invariant of iterated torus knots, preprint, arXiv:0906.3660 (2009).

[Br] E. Brieskorn, Beispiele zur Differenzialtopologie von Singularitäten, Invent. Math. 2 (1966), 1-14.

[COT1] T. Cochran, K. Orr and P. Teichner, Knot concordance, Whitney towers and $L^{2}$-signatures, Ann. of Math. 157 (2003), 433-519.

[COT2] - - - - Structure in the classical knot concordance group, Comment. Math. Helv. 79 (2004), 105-123.

[Co] J. Collins, $L^{2}$ signature of torus knots, preprint (2010), arXiv:1001.1329.

[GLM] C. McA. Gordon, R. Litherland and K. Murasugi, Signatures of covering links, Canad. J. Math. 33 (1981), 381-394.

[HZ] F. Hirzebruch and D. Zagier, The Atiyah-Singer Theorem and Elementary Number Theory, Publish or Perish, Boston, USA, 1974.

[Kau] L. Kauffman, On Knots, Ann. of Math. Stud. 115, Princeton Univ. Press, Princeton, 1987. 
[KM] R. Kirby and P. Melvin, Dedekind sums, $\mu$-invariants, and the signature cocycle, Math. Ann. 29 (1994), 231-267.

[Li] R. A. Litherland, Signatures of iterated torus knots, in: Topology of Low-Dimensional Manifolds (Chelwood Gate, 1977), Lecture Notes in Math. 722, Springer, Berlin, 1979, 71-84.

[Nem] A. Némethi, On the spectrum of curve singularities, in: Singularity (Oberwolfach, 1996), Progr. Math. 162, Birkhäuser, 1998, 93-102.

[NY] M. A. Nouh and A. Yasuhara, Torus knots that cannot be untied by twisting, Rev. Mat. Complut. 14 (2001), 423-437.

[RG] H. Rademacher and E. Grosswald, Dedekind Sums, Carus Math. Monogr. 16, Math. Assoc. Amer., 1972.

[Ro] K. Rosen, Dedekind-Rademacher sums and lattice points in triangles and tetrahedra, Acta Arith. 39 (1981), 59-75.

[Vi] O. Viro, Branched coverings of manifold with boundary and link invariants, I, Math. USSR-Izv. 7 (1973), 1239-1256.

Maciej Borodzik

Institute of Mathematics

University of Warsaw

Banacha 2

02-097 Warsaw, Poland

E-mail: mcboro@mimuw.edu.pl
Krzysztof Oleszkiewicz Institute of Mathematics University of Warsaw Banacha 2 02-097 Warsaw, Poland and

Institute of Mathematics Polish Academy of Sciences Śniadeckich 8

00-956 Warsaw, Poland E-mail: koles@mimuw.edu.pl 